\title{
Schisandrin B Enhances Hepatic/Myocardial Glutathione Regeneration Capacity and Protects Against Oxidant Injury in Rat Livers and Hearts
}

\author{
Pou Kuan Leong ${ }^{\wedge}$, Na Chen ${ }^{\wedge}$ and Kam Ming Ko ${ }^{\wedge} * *$ \\ Division of Life Science, Hong Kong University of Science \& Technology, Clear Water Bay, Hong Kong SAR, China
}

\begin{abstract}
The effects of schisandrin B (Sch B), curcumin, epigallocatechin gallate (EGCG) and resveratrol on the glutathione regeneration capacity (GRC) of liver and heart tissues ex vivo were examined in relation to their protective effects against $\mathrm{CCl}_{4}$ hepatotoxicity in vivo and myocardial ischemia/reperfusion (I/R) injury ex vivo in rats. Results indicated that Sch B, but not curcumin, EGCG or resveratrol, caused hepatic and myocardial GRC enhancement, which was paralleled by its protection against $\mathrm{CCl}_{4}$ hepatotoxicity and myocardial I/R injury. Although resveratrol and curcumin failed to increase the GRC of liver/heart tissues, they did protect against $\mathrm{CCl}_{4}$ hepatotoxicity and myocardial I/R injury. In conclusion, the Sch B-induced enhancement of GRC ex vivo correlated well with its ability to protect tissues against oxidant injury in vivo/ex vivo, suggesting that the GRC assay can be used as a monitor of tissue protection for compounds stimulating glutathione redox cycling. However, phytochemicals can also protect against oxidant injury through various other mechanisms.
\end{abstract}

Keywords: Curcumin, epigallocatechin gallate, glutathione redox cycling, oxidative stress, resveratrol, schisandrin B.

\section{INTRODUCTION}

Oxidative stress, an imbalance in redox status caused by an overproduction and/or inadequate scavenging of reactive oxygen species, was found to be associated with the aging process and a number of pathological conditions [1]. To counteract oxidative stress, reduced glutathione (GSH), the most abundant low molecular weight thiol in mammalian tissues, undergoes non-enzymatic and enzymatic reactions, during which GSH is converted to oxidized glutathione (GSSG). Recent studies demonstrated that a pro-oxidant shift in the GSH/GSSG ratio is associated with age-related cell death, presumably due to the disruption of redox signaling cascades [2]. The maintenance of an optimal GSH/GSSG ratio is therefore critical for cell survival. To maintain the GSH/GSSG ratio under conditions of oxidative stress, cellular GSH can be regenerated from GSSG by a glutathione reductase (GR)-catalyzed reaction and/or be replenished by a two-step de novo synthesis involving glutamate cysteine ligase (GCL) and glutathione synthetase (GS) [3]. The ability of cells/tissues to replenish cellular GSH upon oxidative challenge is referred to as "glutathione recovery capacity" (GRC) [4]. In this connection, pharmacological interventions that can increase the GRC of cells or tissues offer a potential approach to protect against oxidant injury.

Over the past decades, considerable research attention has been focused on the search for phytochemicals capable

\footnotetext{
*Address correspondence to this author at the Division of Life Science, Hong Kong University of Science \& Technology, Clear Water Bay, Hong Kong SAR, China; Tel: +852-23587298; Fax: +852-27198158; E-mail: bcrko@ust.hk

${ }^{\wedge}$ These authors contributed equally to this work.
}

of protecting against oxidant-induced injury. Thus, a growing number of dietary interventions have shown promise as inducers of nuclear factor erythroid 2-related factor 2 (Nrf2, a transcriptional regulator of antioxidant gene expression) that is capable of eliciting an antioxidant response [5]. Schisandrin B (Sch B) is the most abundant dibenzocyclooctadiene lignan isolated from Fructus Schisandrae (FS), a traditional Chinese herb that is clinically prescribed for the treatment of hepatitis. A previous study in our laboratory has demonstrated that treatment with a lignan-riched extract of FS increases the GRC of rat liver tissue homogenates $e x v i$ $v o$, presumably through the enhancement of GR-mediated GSH regeneration [4]. Consistent with this, we have recently shown that the extent of cytoprotection afforded by Sch B correlated with the degree of stimulation in GRC in various types of cultured cells, including AML12 hepatocytes, H9c2 cardiomyocytes and differentiated dopaminergic PC12 neuronal cells [6]. In particular, the resistance of Sch B-preincubated cells to tert-butylhydroperoxide (tBHP)-induced GSH depletion was significantly enhanced [6]. The cytoprotection afforded by Sch B against oxidant injury may be related to its ability to inhibit the depletion of cellular GSH and hence the irreversible process of apoptosis [7].

Recently, the GRC assay ex vivo has been used as a bioactivity monitor for demonstrating the beneficial effect of a nanomized preparation of Sch B as compared with the conventional preparation in rat liver and heart tissues [8]. However, whether or not the enhancement of GRC can result in tissue protection against oxidant injury in vivo/ex vivo has yet to be established. In the present study, we examined the effect of Sch B on the GRC of liver and heart tissues in relation to its protective activity against $\mathrm{CCl}_{4}$-induced toxicity in 
liver and myocardial ischemia/reperfusion (I/R) injury in rats. The effects of curcumin, epigallocatechin gallate (EGCG) and resveratrol were also examined in order to investigate the possible role of glutathione redox cycling in protecting against oxidant injury by these compounds.

\section{MATERIALS AND METHODS}

\section{Chemicals}

Sch B was prepared as previously described [9]. Curcumin, EGCG, tBHP, 5,5'-Dithiobis(2-nitrobenzoic acid) (DTNB), GSH, GSSG, NADPH and NAD were purchased from Sigma Chemical Co. (St. Louis, MO, USA). Resveratrol was obtained from Cayman Chemical Co. (Michigan, USA). All other chemicals were of analytical grade.

\section{Animal Care}

Adult Sprague-Dawley rats (8 to 10 -week-old; 250 to $300 \mathrm{~g}$ ) were maintained under a $12 \mathrm{~h}$ dark/light cycle at an ambient temperature of about $22{ }^{\circ} \mathrm{C}$ and allowed water and food ad libitum in the Animal and Plant Care Facility at the Hong Kong University of Science and Technology (HKUST). Experimental protocols were approved by the Research Practice Committee at the HKUST.

\section{Animal Treatment}

Animals were randomly divided into groups of five animals each. Previous studies have shown that treatment with Sch B $(0.825 \mu \mathrm{mol} / \mathrm{kg} /$ day $)$ for 15 consecutive days induced a glutathione antioxidant response and confer a protective effect in rat hearts [10]. In the study, rats were intragastrically administered Sch B, curcumin, EGCG or resveratrol $(0.825 \mu \mathrm{mol} / \mathrm{kg} / \mathrm{day}, 0.5 \mathrm{~mL}$ per rat) for 15 consecutive days. Control animals were treated with vehicle $(0.5 \mathrm{~mL}$ olive oil $)$ only. Twenty-four hours after the last dosing, the GRC of hepatic and myocardial tissue homogenates was measured. To investigate the effect of Sch B and other phytochemicals on tissue protection in vivolex vivo, rats were subjected to $\mathrm{CCl}_{4}$ hepatotoxicity or myocardial $\mathrm{I} / \mathrm{R}$ injury at the same time point (i.e., $24 \mathrm{~h}$ after the last dosing).

\section{GRC Assay}

Aliquots of $1 \mathrm{~mL}$ pre-warmed (in a water bath at $37^{\circ} \mathrm{C}$ ) liver and heart tissue homogenates were challenged with 1 $\mathrm{mL}$ tBHP (at final concentrations of 0.05 or $0.1 \mathrm{mM}$,) at 37 ${ }^{\circ} \mathrm{C}$ for 60 min. At intervals of $0,1,10,20,30,40,50$ and 60 min, a $200 \mu \mathrm{L}$ aliquot of the reaction mixture was withdrawn and combined with $50 \mu \mathrm{L}$ ice-cold trichloroacetic acid (25\%, $w / v)$. After centrifugation $\left(2,500 \times g\right.$ at $4{ }^{\circ} \mathrm{C}$ for $\left.10 \mathrm{~min}\right)$, the supernatant was analyzed spectrophotometrically for acidsoluble free sulfhydryl group content, an indirect measure of GSH, using DTNB [11]. The GSH levels of samples were determined using a calibration curve. Data were normalized relative to the initial value of GSH (i.e. time $=0$ ) of each sample and were expressed as $\%$ of the initial value. The GRC of each sample was estimated by computing the area under the curve (AUC) of the \% initial value plotted against time, and expressed in arbitrary units.

\section{$\mathrm{CCl}_{4}$ Hepatotoxicity}

Twenty-four hours after treatment with the putative protective agents, rats were given $1 \mathrm{~mL} / \mathrm{kg} \mathrm{CCl}_{4}[10 \%(\mathrm{v} / \mathrm{v})$ in olive oil] orally. Twenty-four hours after $\mathrm{CCl}_{4}$ administration, rats were phenobarbtial-anesthetized and heparinized blood samples were drawn by cardiac puncture, and animals were then killed by cardiac excision. Plasma samples were obtained by centrifuging whole blood samples at 1,500 $\times g$ for $10 \mathrm{~min}$ at $4{ }^{\circ} \mathrm{C}$. The extent of hepatic oxidative damage was assessed by the measurement of plasma sorbitol dehydrogenase (SDH) activity as described previously [9]. The extent of protection against $\mathrm{CCl}_{4}$ hepatotoxicity was estimated using the equation:

$$
\begin{aligned}
& \text { Protection }(\%)=\left[\left(\mathrm{SDH}_{\mathrm{CCl} 4} \text { control }-\mathrm{SDH}_{\text {non-CCl4 control }}\right)-\right. \\
& \left.\left(\mathrm{SDH}_{\mathrm{CCl} 4 \text { drug }}-\mathrm{SDH}_{\text {non-CCl4 drug }}\right)\right] /\left(\mathrm{SDH}_{\mathrm{CCl} 14 \text { control }}-\mathrm{SDH}_{\text {non-CCl4 }}\right. \\
& \text { control }) \times 100 \%
\end{aligned}
$$

\section{Myocardial I/R Injury}

Rat hearts isolated from phytochemical-pretreated rats were subjected to I/R challenge at $24 \mathrm{~h}$ following the last dosing. The excised hearts were retrogradely perfused according to the Langendorff method [12]. Isolated perfused rat hearts were subjected to $40 \mathrm{~min}$ of global ischemia followed by $20 \mathrm{~min}$ of reperfusion. The extent of lactate dehydrogenase (LDH) leakage during the reperfusion period, an indirect index of myocardial injury, was estimated by computing the area under the curve (AUC) of a graph plotting the percent LDH activity (with respect to the mean preischemic value measured during the equilibration period) against reperfusion time (1-20 min), as described previous$1 y,[12]$ and the value was expressed in arbitrary units. The degree of protection against myocardial I/R injury was estimated using the equation:

Protection $(\%)=\left[\left(\mathrm{LDH}_{\mathrm{I} / \mathrm{R}}\right.\right.$ control $-\mathrm{LDH}_{\text {non-I/R }}$ control $)-$ $\left(\mathrm{LDH}_{\mathrm{I} / \mathrm{R}}\right.$ drug $\left.\left.-\mathrm{LDH}_{\text {non-I/R drug }}\right)\right] /\left(\mathrm{LDH}_{\mathrm{I} / \mathrm{R} \text { control }}-\mathrm{LDH}_{\text {non-I/R }}\right.$ control) $\times 100 \%$

\section{Statistical Analysis}

All data were expressed as mean \pm standard error of the mean (SEM), unless otherwise specified. Data of the timedependent change in GSH levels in the GRC assay were analyzed by mixed analysis of variance (mixed ANOVA), while other data were analyzed by one-way analysis of variance (one-way ANOVA). Intergroup differences were detected by the Tukey multiple comparison test, when $p<0.05$.

\section{RESULTS}

Effects of long-term, low dose treatment with Sch B and other phytochemicals on the GRC in rat liver and heart tissues ex vivo

tBHP challenge caused oxidative stress in liver and heart tissues, as evidenced by a rapid depletion of GSH (46\% at 0.25 min post-challenge and $61 \%$ at 10 min post-challenge), 
A Liver

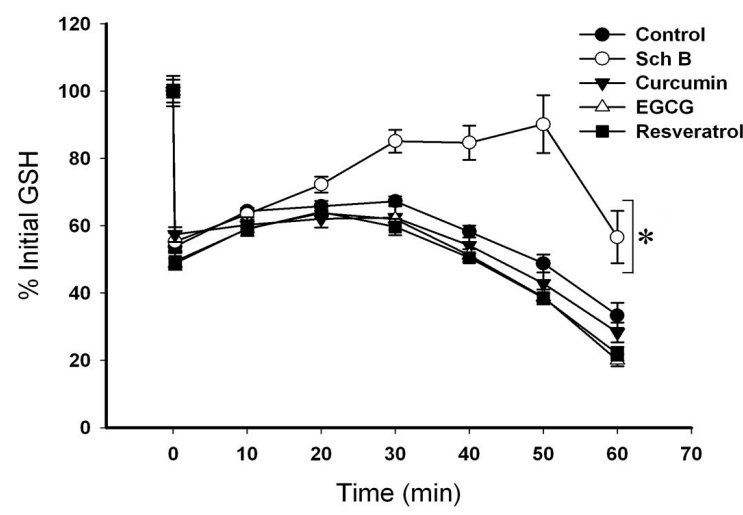

B

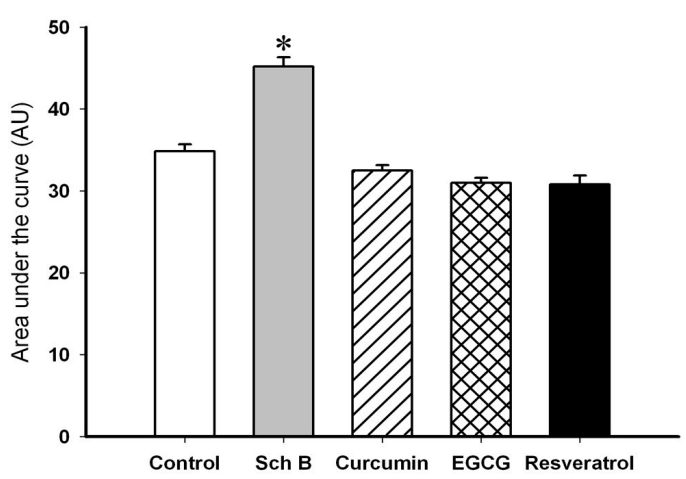

Heart

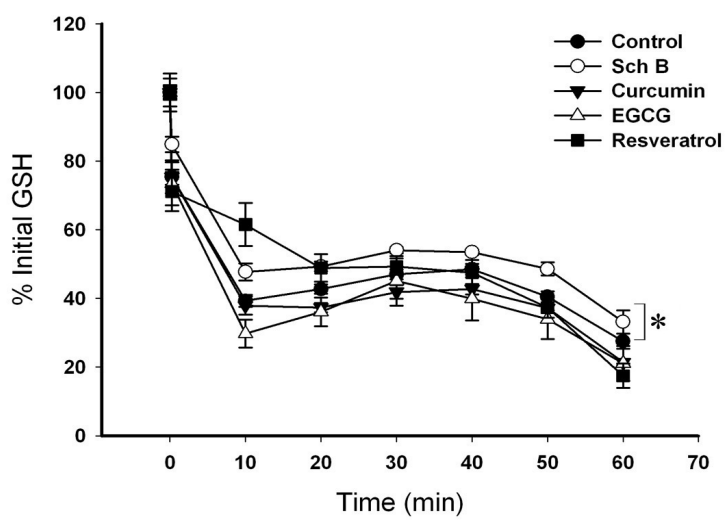

Heart

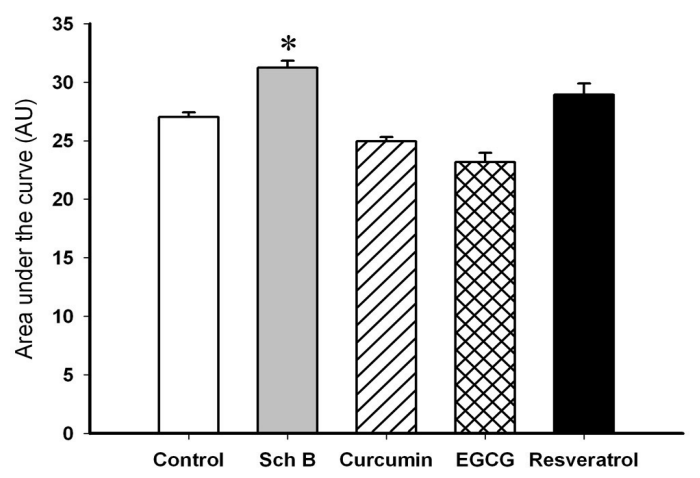

Fig. (1). Effects of long-term, low dose treatment with Sch B and other phytochemicals on glutathione recovery capacity (GRC) in rat liver and heart tissues ex vivo

Rats were intragastrically administered Sch B, curcumin, EGCG or resveratrol $(0.825 \mu \mathrm{mol} / \mathrm{kg} / \mathrm{day})$ for 15 consecutive days. Untreated control mice received oil (vehicle) only. Twenty-four hours after the last dosing, hepatic and myocardial tissue homogenates were challenged with tBHP at 0.1 and $0.05 \mathrm{mM}$ (final concentrations), respectively. Time-dependent changes in GSH levels were monitored during the course of tBHP challenge. Data were expressed as $\%$ initial value by normalizing with initial GSH level (i.e. the value at time $=0$ ) of the respective sample, and were analyzed by mixed analysis of variance (mixed ANOVA) (A). The GRC of each sample was estimated by computing the area under the curve (AUC) obtained by plotting \% initial level against time (min), and expressed in arbitrary units (AU). Then, data were analyzed by one-way analysis of variance (one-way ANOVA) (B). Values given are means \pm SEM, with $n=5$ from two independent experiments. Intergroup differences were detected by Tukey multiple comparison, when $\mathrm{p}<0.05$.

*significantly different from untreated control

when compared with the initial GSH level (Fig. 1). GSH restoration was observed in liver and heart tissues, with the extent of elevation being $67 \%$ (at 30 min post-challenge) and $48 \%$ (at $40 \mathrm{~min}$ post-challenge) of the initial level. Then, GSH levels began to decrease again. Sch B, but not curcumin, EGCG or resveratrol, significantly increased the GRC in liver and heart tissues, with the extent of increase being 30 and $16 \%$, respectively. In contrast, EGCG reduced the GRC in heart tissue by $11 \%$.
Effects of long-term, low dose treatment with Sch B and other phytochemicals on $\mathrm{CCl}_{4}$ hepatotoxicity in vivo and $e x$ vivo myocardial $\mathrm{I} / \mathrm{R}$ injury ex vivo in rats

$\mathrm{CCl}_{4}$ induced oxidative injury in liver, as indicated by the increase in plasma SDH activity ( 7.7 fold), when compared with non- $\mathrm{CCl}_{4}$-challenged controls (Fig. 2). Sch B and resveratrol, but not curcumin or EGCG, protected against 

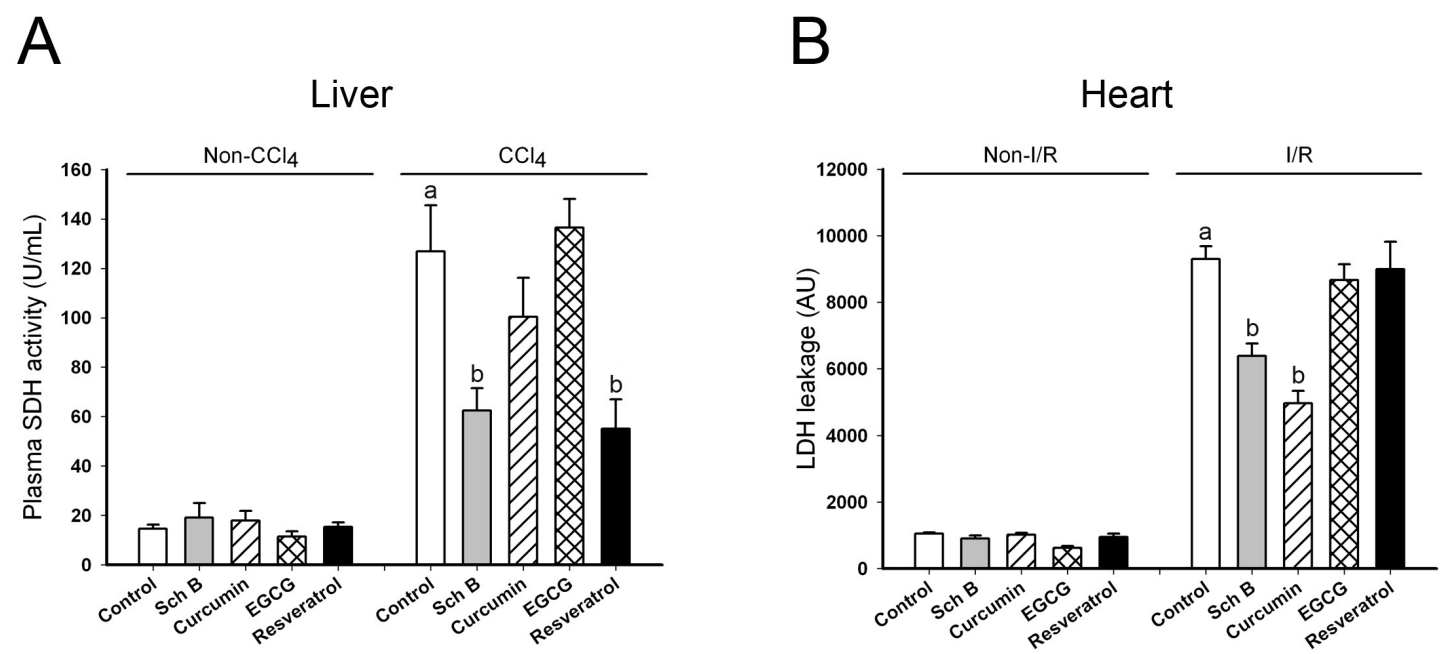

Fig. (2). Effects of long-term, low dose treatment with Sch B and other phytochemicals on $\mathrm{CCl}_{4}$ hepatotoxicity and ex vivo myocardial $\mathrm{I} / \mathrm{R}$ injury in rats

Treatments were performed as described in Fig. 1 . To induce $\mathrm{CCl}_{4}$ hepatotoxicity, rats were orally administered $\mathrm{CCl}_{4}(1 \mathrm{~mL} / \mathrm{kg})$ at 24 hours after the last dosing with phytochemical. At 24 hours post- $\mathrm{CCl}_{4}$ administration, plasma SDH activity was measured (A). To induce myocardial I/R injury, isolated rat hearts were subjected to $40 \mathrm{~min}$ of global ischemia followed by 20 min reperfusion at 24 hours after the last dosing. The extent of myocardial I/R injury was assessed by the measurement of LDH leakage $(\mathbf{B})$. Value given are means \pm SEM, with $n=5$ from two independent experiments. Data were analyzed by one-way analysis of variance (one-way ANOVA), and the intergroup differences were detected by Tukey multiple comparison, when $\mathrm{p}<0.05$.

${ }^{\mathrm{a}}$ significantly different from non- $\mathrm{CCl}_{4}$ control (A) or non-I/R control (B);

bignificantly different from $\mathrm{CCl}_{4}$ control (A) or I/R control (B).

$\mathrm{CCl}_{4}$ hepatotoxicity, with similar degrees of protection (62 and $65 \%$, respectively).

$\mathrm{I} / \mathrm{R}$ produced myocardial oxidative injury, as indicated by an increase in LDH leakage (7.9 fold), when compared with the non-I/R control group (Fig. 2). Sch B and curcumin, but not EGCG or resveratrol, protected against myocardial I/R injury, with the degree of protection afforded by Sch B and curcumin being 34 and $52 \%$, respectively.

\section{DISCUSSION}

Under conditions of oxidative stress, the rapid depletion of GSH can induce irreversible cell death/tissue damage [7]. In this regard, a sustained GRC (a manifestation of GSH replenishment) plays a pivotal role in rescuing cells/tissues from oxidative injury. In the present study, we sought to compare Sch B, curcumin, EGCG and resveratrol, which are inducers of an Nrf2-mediated antioxidant response [5], on the GRC of liver and heart tissues in relation to their hepatoand cardio-protective effects against oxidant-induced injury. It was found that Sch B, but not curcumin, EGCG or resveratrol, enhanced the GRC of liver and heart tissues ex vivo, with the extent of GRC enhancement in the liver being more prominent than that in the heart. The stronger response from the liver may be attributed to higher levels of GSH and glutathione-related enzymes in liver tissue than those in heart tissue [13]. The enhancements in the GRC of liver and heart tissues produced by Sch-B treatment were found to parallel its protective effects against $\mathrm{CCl}_{4}$ hepatotoxicity and myocardial I/R injury. These findings support our hypothesis that the Sch B-induced enhancement of GRC is crucial in rescuing cells from oxidant-induced tissue damage, which is associated with acute GSH depletion and the subsequent initiation of apoptosis.

GR-catalyzed GSH regeneration is important in maintaining an optimal ratio of GSH/GSSG during conditions of oxidative stress. Glucose-6-phosphate dehydrogenase (G6DPH) catalyzes the initial rate-limiting step in the pentose phosphate pathway, which generates the reducing cofactor NADPH, which is required for the GR-catalyzed regeneration of GSH. A cell-based study in our laboratory has found that - of the various phytochemicals investigated only Sch B (but not curcumin, EGCG or resveratrol) increased both GR and G6DPH activities in neuronal cells (unpublished data). This may involve a differential gene expression afforded by different Nrf2-activating phytochemicals. Despite the inability of resveratrol and curcumin to increase the GRC of liver and heart tissues, resveratrol and curcumin were found to protect against $\mathrm{CCl}_{4}$ hepatotoxicity and myocardial $\mathrm{I} / \mathrm{R}$ injury. This observation suggests that glutathione redox cycling may not be the sole protective mechanism against oxidant injury in cells and tissues. The release of pro-inflammatory cytokines, which is triggered by oxidative stress, was demonstrated to accompany liver damage such as that seen fibrosis and cirrhosis [14]. In this connection, a recent study has shown that treatment with 
resveratrol protected against $\mathrm{CCl}_{4}$ hepatotoxicity in rats via the suppression of fibrogenic cytokine release [15]. Similarly, curcumin treatment was found to ameliorate myocardial I/R injury in rats through the inhibition of a toll-like receptor-2-mediated inflammatory response [16].

In conclusion, the Sch B-induced enhancement of GRC ex vivo paralleled with tissue protection against oxidant injury in vivo/ex vivo, suggesting that the GRC assay ex vivo can be used for screening compounds capable of stimulating glutathione redox cycling and thus protect against oxidant injury. However, our results suggest that phytochemicals can also protect against oxidant injury through different mechanisms of action.

\section{REFERENCES}

[1] Calabrese V, Guagliano E, Sapienza M, et al. Redox regulation of cellular stress response in aging and neurodegenerative disorders: role of vitagenes. Neurochem Res 2007; 32: 757-73.

[2] Rebrin I, Sohal RS. Pro-oxidant shift in glutathione redox state during aging. Adv Drug Deliv Rev 2008; 60: 1545-52.

[3] Ballatori N, Krance SM, Notenboom S, et al. Glutathione dysregulation and the etiology and progression of human diseases. Biol Chem 2009; 390: 191-214.

[4] Ko KM, Mak DH, Li PC, et al. Enhancement of hepatic glutathione regeneration capacity by a lignan-enriched extract of Fructus Schisandrae in rats. Jpn J Pharmacol 1995; 69: 439-42.

[5] Kelsey NA, Wilkins HM, Linseman DA. Nutraceutical antioxidants as novel neuroprotective agents. Molecules 2010; 15: 7792-814.
[6] Lam PY, Leong PK, Chen N, Ko KM. Schisandrin B enhances the glutathione redox cycling and protects against oxidant injury in different types of cultured cells. Biofactors 2011; 37: 439-46.

[7] Circu ML, Aw TY. Reactive oxygen species, cellular redox systems, and apoptosis. Free Radic Biol Med 2010; 48: 749-62.

[8] Lau YT, Chen N, Ko KM, Ng KM. Product Design: A Nanomized Nutraceutical with Enhanced Bioactivity and Bioavailability. Ind Eng Chem Res 2012; 51: 7320-6.

[9] Chiu PY, Tang MH, Mak DH, et al. Hepatoprotective mechanism of schisandrin B: role of mitochondrial glutathione antioxidant status and heat shock proteins. Free Radic Biol Med 2003; 35: 368-80

[10] Chiu PY, Leung HY, Poon MK, Ko KM. Chronic schisandrin B treatment improves mitochondrial antioxidant status and tissue heat shock protein production in various tissues of young adult and middle-aged rats. Biogerontology 2006; 7: 199-210.

[11] Godin DV, Garnett ME. Species-related variations in tissue antioxidant status--II. Differences in susceptibility to oxidative challenge. Comp Biochem Physiol B 1992; 103: 743-8.

[12] Ko KM, Yiu HY. Schisandrin B modulates the ischemiareperfusion induced changes in non-enzymatic antioxidant levels in isolated-perfused rat hearts. Mol Cell Biochem 2001; 220: 141-7.

[13] Noeman SA, Hamooda HE, Baalash AA. Biochemical study of oxidative stress markers in the liver, kidney and heart of high fat diet induced obesity in rats. Diabetol Metab Syndr 2011; 3: 17.

[14] Brenner DA. Molecular pathogenesis of liver fibrosis. Trans Am Clin Climatol Assoc 2009; 120: 361-8.

[15] Roy S, Sannigrahi S, Majumdar S, et al. Resveratrol regulates antioxidant status, inhibits cytokine expression and restricts apoptosis in carbon tetrachloride induced rat hepatic injury. Oxid Med Cell Longev 2011; 2011: 703676.

[16] Kim YS, Kwon JS, Cho YK, et al. Curcumin reduces the cardiac ischemia-reperfusion injury: involvement of the toll-like receptor 2 in cardiomyocytes. J Nutr Biochem 2012; 23: 1514-23. 